\title{
Computational Study on Monkey VOR Adaptation and Smooth Pursuit Based on the Parallel Control-Pathway Theory
}

\author{
HIROMITSU TABATA, ${ }^{1,2}$ KENJI YAMAMOTO, ${ }^{3,4}$ AND MITSUO KAWATO ${ }^{1,5}$ \\ ${ }^{1}$ Kawato Dynamic Brain Project, ERATO, Japan Science and Technology Corporation, Kyoto 619-0288; ${ }^{2}$ Nara Institute of \\ Science and Technology, Ikoma-shi 630-0101; ${ }^{3}$ Japan Science and Technology Corporation, Domestic Research Fellow; \\ ${ }^{4}$ National Institute of Advanced Industrial Science and Technology, Ibaraki 305-8568; and ${ }^{5}$ ATR Human Information \\ Science Laboratory, Kyoto 619-0288, Japan
}

Received 12 February 2001; accepted in final form 20 November 2001

Tabata, Hiromitsu, Kenji Yamamoto, and Mitsuo Kawato. Computational study on monkey VOR adaptation and smooth pursuit based on the parallel control-pathway theory. J Neurophysiol 87: 2176-2189, 2002; 10.1152/jn.00168.2001. Much controversy remains about the site of learning and memory for vestibuloocular reflex (VOR) adaptation in spite of numerous previous studies. One possible explanation for VOR adaptation is the flocculus hypothesis, which assumes that this adaptation is caused by synaptic plasticity in the cerebellar cortex. Another hypothesis is the model proposed by Lisberger that assumes that the learning that occurs in both the cerebellar cortex and the vestibular nucleus is necessary for VOR adaptation. Lisberger's model is characterized by a strong positive feedback loop carrying eye velocity information from the vestibular nucleus to the cerebellar cortex. This structure contributes to the maintenance of a smooth pursuit driving command with zero retinal slip during the steady-state phase of smooth pursuit with gain 1 or during the target blink condition. Here, we propose an alternative hypothesis that suggests that the pursuit driving command is maintained in the medial superior temporal (MST) area based on MST firing data during target blink and during ocular following blank, and as a consequence, we assume a much smaller gain for the positive feedback from the vestibular nucleus to the cerebellar cortex. This hypothesis is equivalent to assuming that there are two parallel neural pathways for controlling VOR and smooth pursuit: a main pathway of the semicircular canals to the vestibular nucleus for VOR, and a main pathway of the MST-dorsolateral pontine nuclei (DLPN)_flocculus/ventral paraflocculus to the vestibular nucleus for smooth pursuit. First, we theoretically demonstrate that this parallel control-pathway theory can reproduce the various firing patterns of horizontal gaze velocity Purkinje cells in the flocculus/ventral paraflocculus dependent on VOR in the dark, smooth pursuit, and VOR cancellation as reported in Miles et al. at least equally as well as the gaze velocity theory, which is the basic framework of Lisberger's model. Second, computer simulations based on our hypothesis can stably reproduce neural firing data as well as behavioral data obtained in smooth pursuit, VOR cancellation, and VOR adaptation, even if only plasticity in the cerebellar cortex is assumed. Furthermore, our computer simulation model can reproduce VOR adaptation automatically based on a heterosynaptic interaction model between parallel fiber inputs and climbing fiber inputs. Our results indicate that different assumptions about the site of pursuit driving command maintenance computationally lead to different conclusions about where the learning for VOR adaptation occurs. Finally, we propose behavioral and physiological experiments capable of discriminating between these two possibilities for the site of pursuit

Present address and address for reprint requests: H. Tabata, Kawato Dynamic Brain Project, ERATO, JST, c/o ATR, 2-2 Hikari-dai, Seika-cho, Soraku-gun, Kyoto 619-0288, Japan (E-mail: htabata@ his.atr.co.jp). driving command maintenance and hence for the sites of learning and memory for VOR adaptation.

\section{N T R O D U C T I O N}

The vestibuloocular reflex (VOR) stabilizes images on the retina by causing the eyes to rotate to compensate for head movements. The major circuit achieving horizontal VOR is composed of a three-neuron feedforward control system. Horizontal semicircular canals stimulated by ipsiversive head rotations send signals to the relay neurons in the vestibular nucleus. These relay neurons in turn send signals to the motor neurons of extraocular muscles. Consequently, when the head is rotated to one side, the eyes move in the direction opposite to the head rotation.

Another pathway of the VOR signal contains the flocculus and ventral paraflocculus in the cerebellar cortex. Purkinje cells in the flocculus and ventral paraflocculus have inhibitory projections to the vestibular nucleus. These cells receive two major inputs: parallel fibers and climbing fibers. The activity of the parallel fibers carries visual, vestibular, and eye-movement signals. The climbing fibers project from the inferior olive to the Purkinje cells and cause complex spikes.

In normal monkeys, the gain of VOR, defined as the magnitude of the eye movement velocity divided by the magnitude of the head movement velocity during head turns in darkness, is about 1 . The VOR gain can be adaptively changed. Concretely speaking, when head turns are combined with image motions in the direction opposite to the head turns, adaptation occurs to increase the VOR gain. In contrast, when head turns are combined with image motions in the same direction, adaptation occurs to decrease the VOR gain. VOR adaptation can be experimentally induced by spectacles that either magnify or reduce the scale of vision or by a combination of a rotating chair and a moving visual pattern on a screen.

The flocculus hypothesis (Ito 1970, 1984, 1998) postulates that VOR adaptation is induced by synaptic plasticity in the flocculus guided by error signals conveyed by climbing fiber inputs (Fig. 1A). It postulates that the coincidence of visual climbing-fiber activity and vestibular parallel fiber activity

\footnotetext{
The costs of publication of this article were defrayed in part by the payment of page charges. The article must therefore be hereby marked "advertisement" in accordance with 18 U.S.C. Section 1734 solely to indicate this fact.
} 

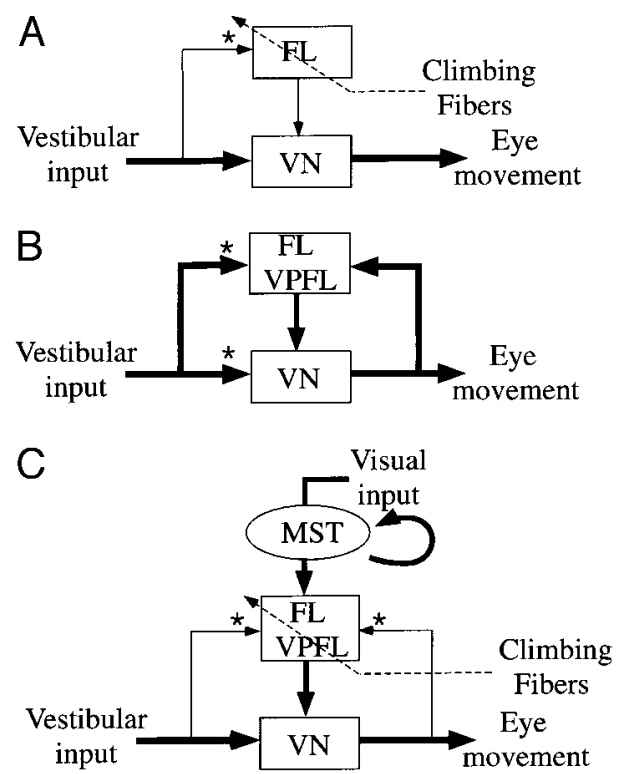

FIG. 1. Specific hypotheses about vestibuloocular reflex (VOR) adaptation. FL, the flocculus; VPFL, the ventral paraflocculus; VN, the vestibular nucleus. $A$ : the flocculus hypothesis. VOR adaptation is induced by synaptic plasticity in the cerebellar cortex when the climbing-fiber activity and vestibular parallelfiber activity coincide. $B$ : the gaze velocity theory leads to the hypothesis that learning is necessary in both the cerebellar cortex and the vestibular nucleus for VOR adaptation. One of the main features is a strong positive feedback from the vestibular nucleus to the cerebellar cortex. $C$ : the parallel controlpathway theory proposed in the present study. Smooth pursuit driving command is maintained in the MST area, so the strength of the positive feedback from the vestibular nucleus to the cerebellar cortex is much weaker than in the model shown in $B$. Asterisk, the site of synaptic plasticity required for VOR adaptation. The thickness of the lines represents the strength of the signal conveyed.

induces long-term depression (LTD) (Ito and Kano 1982) of synapses from the vestibular parallel fibers to the Purkinje cells (Ito 1998). This hypothesis is supported by various experimental data. However, controversy remains about whether or not VOR adaptation can be explained by the synaptic plasticity in the cerebellar cortex alone. In contrast, another major hypothesis postulates that not only the plasticity in the cerebellar cortex but also the learning in the vestibular nucleus are necessary for VOR adaptation (Lisberger 1988, 1994; Lisberger and Sejnowski 1992; Miles and Lisberger 1981) (Fig. 1B). One main reason why the preceding controversy has continued for around $20 \mathrm{yr}$ appears to be that neurophysiological experiments have not been able to directly measure the changes in the synaptic weights of the vestibular parallel fiber inputs to the Purkinje cells associated with VOR adaptation. Accordingly, it has been extremely difficult to determine the sites of learning by only experimental procedures. To resolve this controversy, it seems inevitable that we may have to integrate various experimental data through theoretical studies including computer simulations.

The Lisberger (1994) model was designed based on detailed physiological data (Lisberger et al. 1994a-c) involving not only VOR but also VOR cancellation and smooth pursuit. VOR cancellation is caused by tracking a moving target paired with equivalent head turns. Primates use smooth-pursuit eye movements to accurately track a slow moving target. The Lisberger (1994) model supports the idea that the learning in both the cerebellar cortex and vestibular nucleus is necessary for VOR adaptation. On the other hand, Fujita (1982a,b) and Kawato and Gomi (1992), for example, have come to support the flocculus hypothesis on theoretical grounds. These models, however, do not reproduce smooth-pursuit eye movements or VOR cancellation. In the monkey, the Purkinje cells in the flocculus and ventral paraflocculus are activated not only during VOR but also during smooth pursuit and VOR cancellation (Miles et al. 1980b). Although the Lisberger (1994) model explains how the VOR pathways are used for smooth pursuit and VOR cancellation, this is not explained by the Fujita (1982a,b) or Kawato and Gomi (1992) models. Furthermore, the latter models do not reproduce detailed firing data like Lisberger's (1994) model.

Lisberger's (1994) model is characterized by a strong positive feedback loop from the vestibular nucleus to the cerebellar cortex. This structure plays an essential role in reproducing both VOR adaptation and smooth pursuit (Lisberger 1994; Lisberger and Sejnowski 1992). In the present paper, we first propose a conceptual hypothesis that suggests that the pursuit driving command is maintained in the medial superior temporal (MST) area (Maunsell and Van Essen 1983) for zeroretinal-slip smooth pursuit or the target blink condition based on previous firing data of the MST area and also on physiological data of the ocular following response (OFR) (Miles et al. 1986), which is caused by the movements of large visual scenes. Second, we theoretically show how our hypothesis can explain physiological data during VOR in the dark, VOR cancellation, smooth pursuit, and OFR. Then, we modify Lisberger's (1994) computer-simulation model based on this theory and attempt to reproduce physiological data identical to Lisberger's (1994) model. We also conduct additional simulation experiments showing the possibility that VOR adaptation can be reproduced with the learning site in the cerebellar cortex alone while incorporating detailed models of the synaptic plasticity. Finally, we propose behavioral and physiological experiments that can test the predictions of our hypothesis.

\section{PARALLEL CONTROL-PATHWA Y THEORY}

\section{New hypothesis for the maintenance and cancellation of} slow eye movements

Physiological experiments have shown that the MT (middle temporal) area and the MST area in the superior temporal sulcus are related to the generation of smooth-pursuit eye movements (Erickson and Dow 1989; Groh et al. 1997; Komatsu and Wurtz 1989; Sakata et al. 1983; Thier and Erickson 1992). Both of these areas project to the dorsolateral pontine nuclei (DLPN) (Boussaoud et al. 1992; Brodal 1978; Glickstein et al. 1980, 1985; May and Andersen 1986; Tusa and Ungerleider 1988; Ungerleider et al. 1984), which in turn project to the cerebellum (Brodal 1979, 1982; Langer et al. 1985). The mossy fibers in the ventral paraflocculus have similar firing characteristics to DLPN and MST neurons (Kawano and Shidara 1993). In Lisberger's (1994) model, it is assumed that the smooth-pursuit system and VOR system jointly utilize the flocculus/ventral paraflocculus of the cerebellar cortex and their downstream circuit.

The pursuit system receives retinal slip signals as its inputs. Even when the target is stabilized at the fovea to make the retinal slip signals negligible (target-stabilization), ongoing 
smooth pursuit is nearly maintained in monkeys (Morris and Lisberger 1987). The pursuit movement gradually drops its velocity with a time constant of 400-800 ms in humans (Pola and Wyatt 1997). Furthermore, when the visual target suddenly vanishes (pursuit blink), the smooth eye velocity does not rapidly disappear in the monkeys (Kawano et al. 1994; Newsome et al. 1988; Sakata et al. 1983). The smooth eye velocity decreases with a time constant of about $100 \mathrm{~ms}$ but does not abruptly drop to zero for 400-500 ms in human subjects who do not expect the target to reappear (Pola and Wyatt 1997). In human subjects who expect the target to reappear, about $40-$ $60 \%$ of the smooth eye velocity is maintained (Becker and Fuchs 1985). These findings indicate that there must be a mechanism that maintains the pursuit driving command without retinal slip signals, although the site of such a mechanism remains unknown. In Lisberger's (1994) model, it is suggested that the strong positive feedback loop from the vestibular nucleus to the cerebellar cortex works as a mechanism to maintain eye velocity during the steady-state phase of smooth pursuit.

Here, we propose the MST area (Fig. 1C) as another possible site of the maintenance mechanism for the pursuit driving command for the following reasons. During target-stabilization, the activity of the MT area, which has projections to the MST area (Maunsell and Van Essen 1983; Ungerleider and Maunsell 1986), rapidly decreased (Newsome et al. 1988). In contrast, the activity of many neurons in the dorsal-medial portion of the MST area (MSTd) and the activity of some in the lateral-anterior portion of the MST area (MSTl) in monkeys were maintained (Newsome et al. 1988). Furthermore, during pursuit blink, the activity of the MT area rapidly decreased (Newsome et al. 1988). However, some MST neurons in monkeys maintained their activity, although there was a moderate decrease (Kawano et al. 1994; Newsome et al. 1988; Sakata et al. 1983). These findings indicate that during the steady-state phase of smooth pursuit, many neurons in the MST area maintain their activity independent of the input of retinal slip signals, which is represented in the MT area.

Some neurons in the MST area (Kawano et al. 1994), DLPN (Kawano et al. 1992), and ventral paraflocculus in the cerebellar cortex (Shidara and Kawano 1993) increase firing rates not only during smooth pursuit but also during OFR. Chemical lesions in the MST area produce a decrement in ipsilateral and vertical OFR (Shidara et al. 1991; Takemura et al. 2000). Furthermore, lesions in the MST area (Dürsteler and Wurtz 1988), DLPN (May et al. 1988), and flocculus/ventral paraflocculus (Zee et al. 1981) cause deficits in both smooth pursuit and optokinetic nystagmus (OKN). OKN is also caused by movements of a large visual scene as is OFR. These phenomena indicate that at least some neurons in the MST area, DLPN, and the flocculus/ventral paraflocculus, are used to control both smooth pursuit and OFR (Kawano 1999). In the present paper, therefore we assume that smooth pursuit and OFR share some common neural circuits that include the MST area, DLPN, and the downstream of the flocculus and ventral paraflocculus. However, the results of a visual stimulus elimination experiment during OFR (OFR blank) markedly differed from the results during pursuit blink. A momentary elimination of the OFR stimulus rapidly diminished the activity of DLPN neurons, and eye velocity dropped to zero in about $60 \mathrm{~ms}$ when a blurred random-dot pattern was used (Kawano et al. 1992). The similar stimulus (OFR blank) also reduces rapidly the activity of MST neurons (examined in 6 neurons by K. Kawano and M. Shidara, personal communication).

These results imply that the neural activity in the MST area is closely correlated to eye movements in pursuit blink and OFR blank experiments. Based on these results, we propose that the neural activity of the MST area is the most critical source for maintaining smooth-pursuit eye movements. We assume the neural activity in the MST area represents an estimated target velocity even when the retinal slip signals are small or when the target of pursuit briefly disappears. We will later discuss the possible neural mechanisms of this firing maintenance function, which does not work for the OFR blank.

Based on this hypothesis, we propose a new theory that can explain data on VOR, VOR cancellation, smooth pursuit, and OFR (Fig. 1C). Under our schema, the pursuit driving command is maintained in the MST area, so we assume that the positive feedback loop from the vestibular nucleus to the cerebellar cortex plays a much smaller role than in Lisberger's model. Therefore the major input to the cerebellar cortex during sustained pursuit is not eye-velocity feedback but the estimated target-velocity signal from the MST area. In other words, we propose that these eye-movement systems contain two major parallel control pathways. The first pathway includes the MT/MST-DLPN-flocculus and ventral paraflocculus-vestibular nucleus-extraocular muscles. The second pathway includes the semicircular canal-vestibular nucleus-extraocular muscles. We postulate that smooth pursuit and OFR are mainly generated through the first pathway, that VOR is mainly generated through the second pathway, and that VOR cancellation utilizes both pathways in combination. Therefore we name the new schema proposed in the present paper the "parallel control-pathway theory." In the next section, we will describe how our schema can conceptually explain behavioral and physiological data based on comparison with another theory.

\section{Comparison of the parallel control-pathway theory with the gaze-velocity theory}

Miles et al. (1980b) showed that the discharge of many Purkinje cells in monkeys during VOR, VOR cancellation, and smooth pursuit encodes the "horizontal gaze velocity," which is defined as the sum of the velocity of the eyes with respect to the head and the velocity of the head with respect to the world. This hypothesis, called the gaze-velocity theory, can explain physiological data during VOR in the dark, VOR cancellation, and smooth pursuit. Gaze-velocity Purkinje cells exhibit strong activity during VOR cancellation and smooth pursuit but only weak activity during VOR in the dark. The interpretation of this by the authors is that gaze-velocity Purkinje cells receive head-movement information and eye-movement information through feedback loops. First of all, during VOR these two inputs cancel each other (Fig. 2A). Second, during VOR cancellation, the activity of these cells purely corresponds to vestibular inputs, i.e., head-movement information, because the eye velocity is zero (Fig. $2 B$ ). Third, the smooth pursuit signal is maintained in the positive feedback loop from the vestibular nucleus to the cerebellar cortex (Fig. 2C). Consequently, Purkinje cells exhibit strong activity during smooth pursuit. 


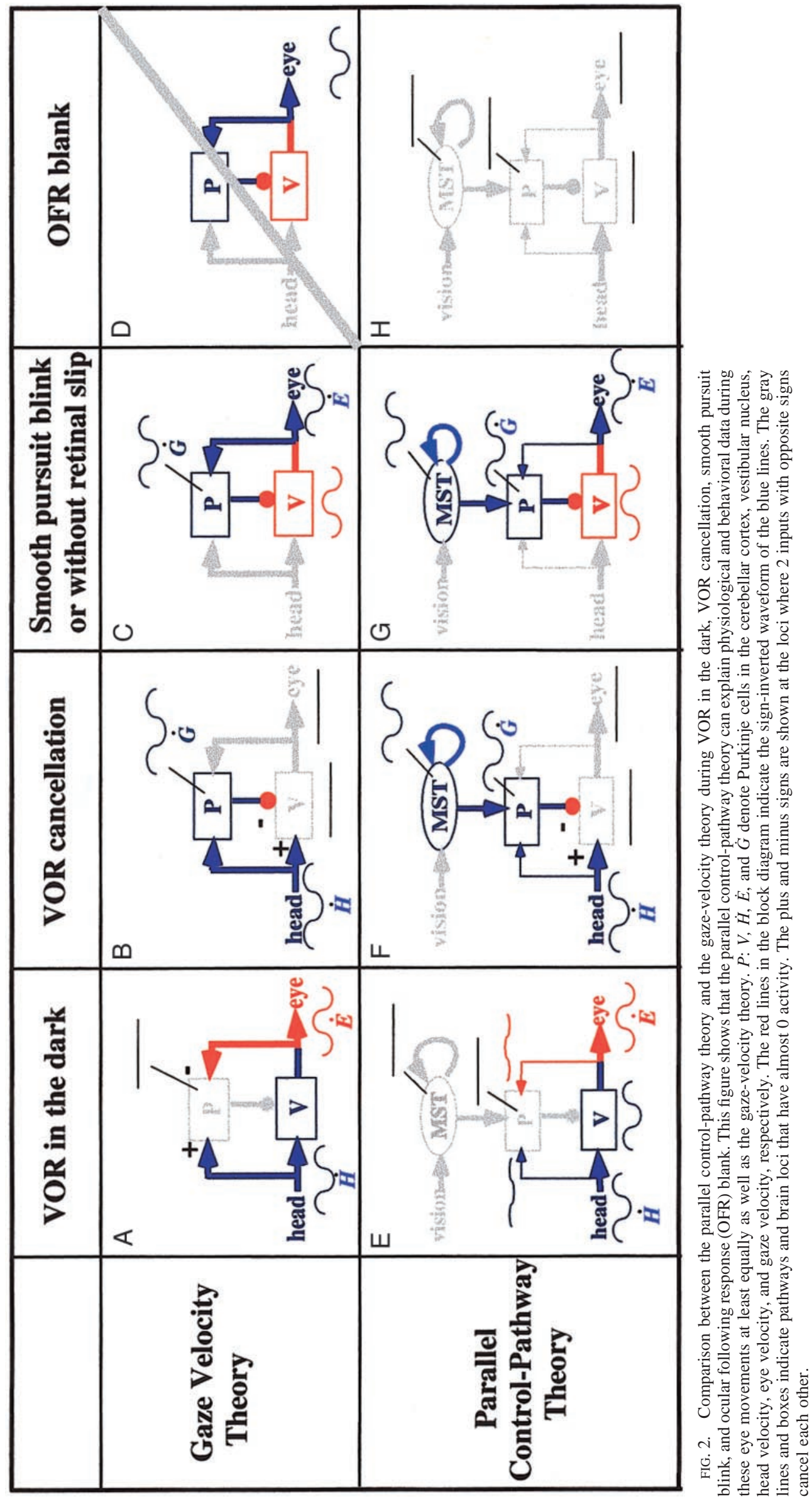


One problem with the gaze velocity theory is as follows. Because the neural circuits for pursuit and OFR overlap downstream from the cerebellar cortex, the OFR should be maintained by the strong positive feedback connection from the vestibular nucleus to the cerebellar cortex even with a sudden elimination of the visual stimulus (Fig. 2D). However, a sudden elimination of the visual stimulus during OFR will rapidly decrease the eye movements (Kawano et al. 1992) as already explained. Here, we note that OFR is outside the scope of the gaze velocity theory. The prediction shown in Fig. $2 D$ is a model that we produced by extrapolating the theory framework.

In the parallel control-pathway theory, the major pursuit driving command maintenance mechanism is located within the MST area. Thus we can assume a much smaller gain for the positive feedback loop, that is, much weaker eye-velocity inputs as well as much weaker vestibular inputs to the cerebellar cortex. Therefore Purkinje cells in the flocculus and ventral paraflocculus can come to show little or no modulation during VOR in the dark when the VOR gain is normal (Fig. $2 E$ ). Control of VOR cancellation activates the neural mechanism for smooth pursuit because the subject must track a moving target with coinciding head movements. During VOR cancellation, therefore the cerebellar cortex receives visually driven inputs from the MST area (Fig. $2 F$ ). Because of this, Purkinje cells exhibit vigorous activity during VOR cancellation. We postulate that the eyes do not move in the steady-state phase because the vestibular input and the signal from the cerebellar cortex, which is mainly composed of the estimated target velocity, cancel each other at the vestibular nucleus. This possibility has already been suggested by Ito (1993). The Purkinje cells exhibit strong activity during smooth pursuit, even if the positive feedback loop from the vestibular nucleus to the cerebellar cortex is no longer strong, because the pursuit driving command is maintained mainly in the MST area when the retinal slip signals are eliminated (Fig. 2G). Furthermore, under our assumption, both MST activity and eye movement rapidly diminish during an OFR blank (Fig. $2 H$ ) because the mechanism maintaining the target velocity, even without visual inputs in the MST area, does not work (see DISCUSSION: Site of the feedback loop maintaining the pursuit driving command).

\section{Mathematical examination of the parallel control-pathway and gaze-velocity theories}

The preceding conceptual investigation indicates that our theory can explain the data on Purkinje cell activity in Miles et al. (1980b) at least equally as well as the gaze-velocity theory. Here, we provide a mathematical foundation for this qualitative argument by comparing the parallel control-pathway theory and the gaze-velocity theory.

First, we mathematically explain the gaze velocity theory. When the VOR gain is 1, eye velocity $\dot{E}$ has the same amplitude as head velocity $\dot{H}$, and the opposite sign during VOR in the dark

$$
\dot{E}=-\dot{H}
$$

In the gaze-velocity theory, the activity of Purkinje cells $P$ is almost zero during VOR in the dark because the strong eyevelocity feedback and strong vestibular inputs cancel each other (Fig. 2A). During VOR cancellation, the eye velocity is zero $(\dot{E}=0)$ because strong inhibitory signals from the Purkinje cells ( $P$ of Fig. $2 B$ ) cancel the vestibular inputs at the vestibular nucleus ( $V$ of Fig. $2 B$ ). Gaze velocity $\dot{G}$ is defined as the summation of head velocity $\dot{H}$ and eye velocity $\dot{E}$

$$
\dot{G}=\dot{H}+\dot{E}
$$

During VOR cancellation, moreover, gaze velocity $\dot{G}$ is the same as head velocity $\dot{H}$ because eye velocity $\dot{E}$ is zero. Therefore

$$
P=\dot{H}=\dot{G}
$$

Consequently, in the gaze-velocity theory, the strong activity of Purkinje cells $P$ under VOR cancellation, that is, gaze velocity $\dot{G}$, corresponds to the purely vestibular input $\dot{H}$ (Fig. 2B).

Next, we mathematically explain the parallel control-pathway theory. Here, eye velocity $\dot{E}$ during VOR in the dark, VOR cancellation, and smooth pursuit is composed of two parallel velocity components relative to vestibular input $\left(\dot{E}_{\text {vestibular }}\right)$ and to visual input $\left(\dot{E}_{\text {visual }}\right)$

$$
\dot{E}=\dot{E}_{\text {vestibular }}+\dot{E}_{\text {visual }}
$$

During VOR in the dark (Fig. $2 E$ ), this equation becomes as follows

$$
\dot{E}=\dot{E}_{\text {vestibular }}=-\dot{H}
$$

During VOR cancellation, visual-related input $\left(\dot{E}_{\text {visual }}\right)$ and vestibular input ( $\left.\dot{E}_{\text {vestibular }}\right)$ cancel each other at the vestibular nucleus shown by $V$ in Fig. $2 F$ so that the eye velocity maintains a zero value $(\dot{E}=0)$. Therefore

$$
\dot{E}_{\text {visual }}=-\dot{E}_{\text {vestibular }}
$$

The Purkinje cell activity during the VOR cancellation is induced by visual-related input $\left(\dot{E}_{\text {visual }}\right)$ generated in the MST area. This assumption is supported by the physiological data that the MST neurons are excited during VOR cancellation (Kawano et al. 1984). Therefore

$$
P=\dot{E}_{\text {visual }}=-\dot{E}_{\text {vestibular }}=\dot{H}=\dot{G}
$$

Equation 7 is the same as Eq. 3 derived by the gaze-velocity theory. In other words, mathematically speaking, both theories can equally well explain the physiological data of the Purkinje cells shown in Miles et al. (1980b). However, these two theories produce quite different predictions about the strengths of vestibular and eye-velocity feedback inputs to Purkinje cells. Furthermore, as we show in the next section, our theory predicts a different learning site from those of the gaze velocity theory.

COMPUTER SIMULATION OF SMOOTH PURSUIT AND VOR ADAPTATION

\section{Model architecture}

In the simulations of the present paper, we use a new simulation model modified from the model of Lisberger 


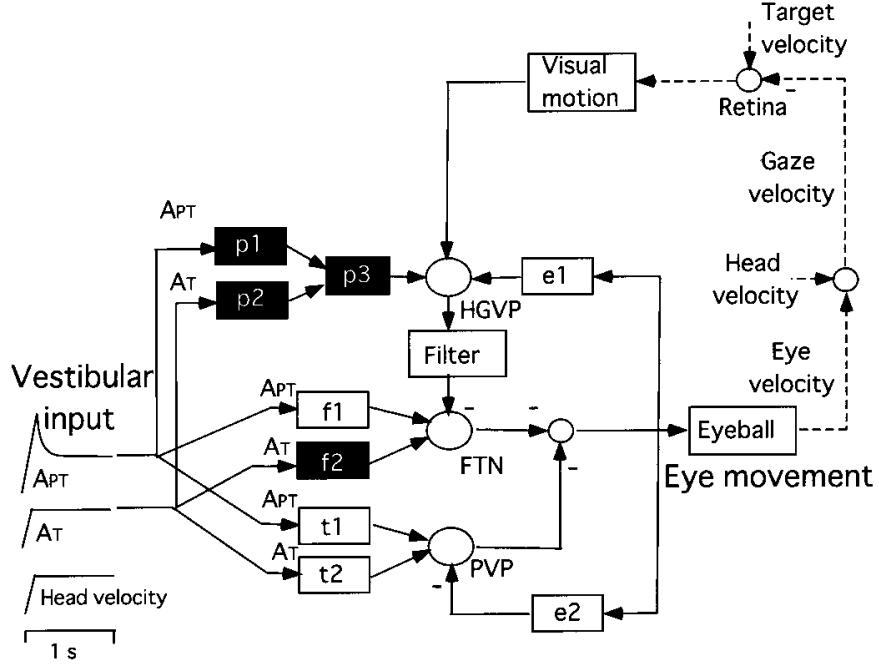

FIG. 3. The simulation model used in Lisberger (1994). This model can simulate smooth pursuit with the head stationary and VOR evoked by rapid changes in the head velocity. p1, p2, p3, f1, f2, t1, t2, e2, and e1 denote the signal-transduction strength of each signal pathway. The values of $\mathrm{f} 1, \mathrm{t} 1, \mathrm{t} 2, \mathrm{e} 2$, and $\mathrm{e} 1$ are set to $0,0.15,0.35,0.175$, and 0.825 , respectively. The black colored boxes p1, p2, p3, and f2, change their values for each VOR gain. These values are set as follows for VOR gains of $1.6,1$, and 0.4 , respectively: $\mathrm{p} 1=$ $0.04,0.325,0.76 ; \mathrm{p} 2=0.96,0.675,0.24 ; \mathrm{p} 3=1.22,1.0,0.75$; and $\mathrm{f} 2=0.72$, $0.5,0.25$. The transfer function of the box labeled "filter" is $1 /(0.1 s+1)$ in a Laplace expression, and its time constant is $100 \mathrm{~ms}$. An 8-ms time delay is set in the section between the eye velocity and gaze velocity, and a 65-ms time delay is set in the section between the retina and visual motion. Here, $A_{\mathrm{PT}}$ and $A_{\mathrm{T}}$ correspond to the vestibular inputs, phasic-tonic responses, and tonic responses that the model receives. We do not use the visual inputs to horizontal gaze-velocity Purkinje cells (HGVP) during the simulation of VOR in the dark. During the simulation of smooth pursuit, the gain of the visual inputs to HGVP is 0.11. A notable feature of this model is the positive feedback loop with a total gain of 1, which is composed of both the feedback to HGVP (e1) and the feedback to PVP (e2).

(1994). To begin with, therefore we will explain the architecture of Lisberger's (1994) model. Figure 3 illustrates Lisberger's (1994) model, which is based on the schema represented in Fig. $1 B$. The output from the model is the eye velocity, and the inputs are the head velocity and target velocity. When the head velocity is given as a step-like function, the model receives two different vestibular inputs: tonic $\left(A_{\mathrm{T}}\right)$ and phasictonic $\left(A_{\mathrm{PT}}\right)$ responses (Lisberger and Pavelko 1986). These vestibular inputs are conveyed to the horizontal gaze-velocity Purkinje cells (HGVP) in the flocculus and ventral paraflocculus and to the flocculus target neurons (FTN) and position vestibular pause cells (PVP) in the vestibular nucleus. Visual inputs to the model originate at the summing junction labeled "retina" and are calculated from the difference between the target velocity and the gaze velocity. These visual inputs are conveyed to the HGVP via the box labeled "visual motion," which implements the smooth-pursuit model proposed in Krauzlis and Lisberger (1989). In the box labeled "visual motion," the retinal signals are processed in three parallel pathways, which are related to the image velocity, the image acceleration, and the motion transient. These visual-related inputs are conveyed to HGVP. The parameters, p1, p2, p3, f1, $\mathrm{f} 2$, $\mathrm{t} 1, \mathrm{t} 2$, e1, and e2 described in Fig. 3 denote the signaltransduction strength of each pathway. These values are given in the description of Fig. 3 and were set to allow each node to reproduce physiological data quantitatively. This model successfully reproduces both the physiological and behavioral data obtained in VOR in the dark, smooth pursuit, and VOR cancellation.

The major features of this model are as follows. First, the positive feedback loop from the vestibular nucleus to the cerebellar cortex is strong to cancel the vestibular inputs to HGVP during VOR in the dark when the VOR gain is normal. The total gain of the positive feedback loop is 1 because it is composed of both the feedback from the vestibular nucleus to the cerebellar cortex $(\mathrm{e} 1=0.825)$ and the feedback to the PVP $(\mathrm{e} 2=0.175)$. Accordingly, the eye-velocity signal can be maintained perfectly in this positive feedback loop without any extra inputs from the outside. Second, the strength of the vestibular inputs to HGVP is the same as that of the inputs to the vestibular nucleus $(\mathrm{p} 3=\mathrm{f} 1+\mathrm{f} 2+\mathrm{t} 1+\mathrm{t} 2)$ so that they cancel out at the vestibular nucleus level. Third, the learning sites are both in the cerebellar cortex (p1, p2, p3) and in the vestibular nucleus (f2), which are shown as boxes with blackand-white reversed.

Figure 4 illustrates the circuit used in the present simulation that implements the parallel control-pathway theory represented in Fig. 1C. The principal differences from Lisberger's model are the following three points. First, the target velocity signal during smooth pursuit is maintained upstream to the Purkinje cells so that the feedback loop from the vestibular nucleus to the cerebellar cortex is much weaker $(\mathrm{e} 1=0.175)$ than in Lisberger's model. Second, the vestibular inputs to the cerebellar cortex are also much weaker than the vestibular inputs to the vestibular nucleus $(\mathrm{p} 1+\mathrm{p} 2<\mathrm{f} 1+\mathrm{f} 2+\mathrm{t} 1+\mathrm{t} 2)$.

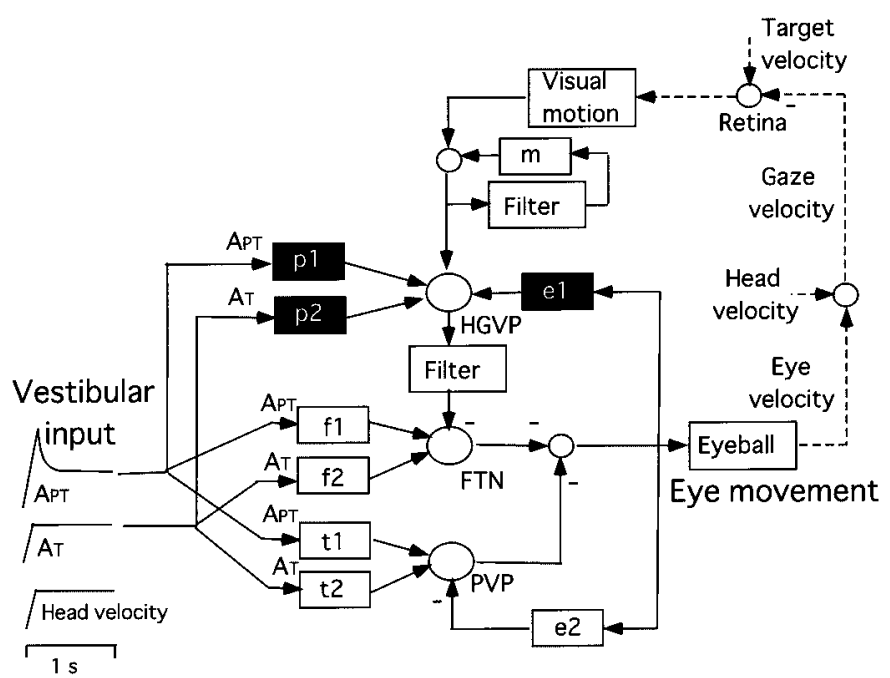

FIG. 4. New VOR adaptation model implementing the parallel controlpathway theory proposed in Fig. $1 C$. The basic structure is similar to that of the model shown in Fig. 3. The major differences from the model shown in Fig. 3 are as follows. First, the positive feedback loop downstream of HGVP has a total gain of $0.35(\mathrm{e} 2=\mathrm{e} 1=0.175)$ instead of 1 . Second, the pursuit signal is maintained at the site assumed to be the MST area $(\mathrm{m}=$ 0.95). Third, the only learning site of VOR adaptation is the cerebellar cortex (p1, p2, e1). We set the values of $\mathrm{f} 1, \mathrm{f} 2, \mathrm{t} 1, \mathrm{t} 2$, and $\mathrm{m}$ to $0,0.5,0.15$, 0.35 , and 0.95 , respectively. The black colored boxes $\mathrm{p} 1, \mathrm{p} 2$, and e1, correspond to sites of plasticity. We set p1 $=-0.004,0.330,0.696, \mathrm{p} 2=$ $-0.02,0.02,0.04$, and $\mathrm{e} 1=0.185,0.175,0.165$ for VOR gains $1.6,1$, and 0.4 , respectively. The transfer function of the box labeled "Filter" is $1 /(0.1 s+1)$ in a Laplace expression, and its time constant is $100 \mathrm{~ms}$. The other details are the same as in Lisberger's model. 
Third, we assume the learning site to be only in the cerebellar cortex (p1, p2, e1).

We use a 50-ms duration ramp with a head velocity increasing from 0 to $30^{\circ} / \mathrm{s}$ followed by a head motion of $1 \mathrm{~s}$ at $30 \%$ as a vestibular stimulus. We set the values of $\mathrm{p} 1$ and $\mathrm{p} 2$ to 0.33 and 0.02 , respectively, when the VOR gain is normal. Then, we set the values of $\mathrm{f} 1, \mathrm{f} 2, \mathrm{t} 1, \mathrm{t} 2$, and $\mathrm{m}$ to $0,0.5,0.15,0.35$, and 0.95 , respectively, regardless of the VOR gain.

MATLAB/SIMULINK (MathWorks) was used on a Sun workstation.

\section{Simulation results of smooth pursuit and VOR cancellation}

First of all, we investigate whether or not our model can reproduce smooth pursuit and VOR cancellation equally as well as Lisberger's model because we change the site of the positive feedback loop maintaining the pursuit driving command. Figure $5 \mathrm{~A}$ shows the simulation results of our new model for smooth pursuit. The neural activity of HGVP, FTN, and PVP, and eye movement are shown when a visual target moves in a step-ramp at $20 \%$ s. The $\cdots$ in the eye movement of Fig. 5A corresponds to the target velocity. Lisberger (1994) showed that his model can successfully reproduce physiological data. Consequently, for comparison, we also show simulation results when we use the Lisberger (1994) model, represented by - - in Fig. 5A. Because the simulation results of our model are almost the same as those of Lisberger's model, we can say that our model can also reproduce smooth-pursuit eye movements. VOR cancellation was also reproduced by our new model (Fig. 5B). Consequently, a simple positive feedback loop within the MST area in our model can maintain targetvelocity information under the zero retinal slip condition in the steady-state phase of smooth pursuit and VOR cancellation.

\section{Simulation results of VOR adaptation}

The VOR adaptation simulation by Lisberger's (1994) model showed unstable runaway under the assumption of learning only in the cerebellar cortex. Accordingly, Lisberger (1994) claimed that learning was necessary in both the vestibular nucleus and the cerebellar cortex. The unstable behaviors of Lisberger's (1994) model are attributed to the assumption that the positive feedback downstream of the cerebellar cortex has a gain of 1 . The gain 1 positive feedback mathematically implies metastable dynamics and infinitely small perturbations can make the system unstable. On the other hand, our model is more stable because we have significantly reduced the positive feedback gain to 0.35 . Therefore our model offers the possibility of reproducing VOR adaptation with learning only in the cerebellar cortex.

To test this possibility, we performed simulations of VOR adaptation with the learning site only in the cerebellar cortex. We assumed that the synaptic efficacy changes occurred at $\mathrm{p} 1$, $\mathrm{p} 2$, and e1. We set $\mathrm{p} 1=-0.004,0.330,0.696 ; \mathrm{p} 2=-0.02$, $0.02,0.04$; and $\mathrm{e} 1=0.185,0.175,0.165$ for VOR gains of 1.6, 1 , and 0.4 , respectively. Figure $6 A$ shows the simulation results of VOR in the dark. This figure shows the neural activity of
HGVP, FTN, and PVP and eye movement of VOR in the dark for three conditions of the VOR gain: low, normal, and high. The - - - - - and - - - correspond to low, normal, and high gains, respectively. For comparison, we also show the simulation results of Lisberger (1994) in Fig. 6B. The results shown in Fig. $6 A$ are almost the same as those shown in $B$. Our new model can therefore reproduce VOR adaptation stably based on learning in the cerebellar cortex alone. This similarity of simulation results by the two models demonstrates that the conclusion in Lisberger (1994), that two learning sites are necessary to reproduce VOR adaptation stably, is the direct consequence of assuming that the positive feedback loop gain from the vestibular nucleus to the cerebellar cortex is 1 .

Lisberger (1994) stated that learning for VOR adaptation has no influence on smooth pursuit behavior. In our model, one of the learning sites is $\mathrm{e} 1$, which is included in a smooth pursuit pathway. However, the simulation results for smooth pursuit exhibited very little influence from VOR adaptation. The change in smooth pursuit gain was only 0.011 and 0.008 for the VOR gains of 1.6 and 0.4 , respectively.

\section{Learning simulation}

In the preceding simulation of VOR adaptation, we set the different values of synaptic weights for different VOR gains heuristically to reproduce firing and behavioral data changes just like Lisberger (1994). In Lisberger's (1994) model, these manual adjustments of synaptic weights seem inevitable to reproduce VOR adaptation because synaptic weights must change in a complicated and irregular manner (i.e., p1 increased, $\mathrm{p} 2$ decreased, $\mathrm{p} 3$ decreased, e1 no change, and only f2 decreased while $\mathrm{f} 1, \mathrm{t} 1, \mathrm{t} 2$ had no change, when VOR gain decreased). It would be difficult to explain these synaptic weight changes through any known synaptic plasticity of Purkinje cells or by unknown synaptic plasticity in the vestibular nucleus. On the other hand, in our new model, the pattern of synaptic efficacy changes was simple and regular (i.e., p1 increased, $\mathrm{p} 2$ increased for VOR gain decreased and no change in the vestibular nucleus). Thus we expected that this could easily be reproduced by the known synaptic plasticity of Purkinje cells. Below, we show how our model can automatically reproduce VOR adaptation by heterosynaptic interaction between parallel fiber inputs and climbing fiber inputs with the synaptic plasticity rules known in the cerebellar cortex. We also attempt to show quantitatively that the synaptic weight changes assumed in the preceding simulations are biologically plausible.

The physiologically demonstrated synaptic plasticity of Purkinje cells is as follows. If the parallel-fiber activity coincides with the climbing-fiber activity, LTD is induced in the synapses from parallel fibers to the Purkinje cells in the cerebellar cortex (Ito and Kano 1982; Ito et al. 1982). If the parallel-fiber activity does not coincide with the climbing-fiber activity, long-term potentiation (LTP) is induced (Hirano 1990; Sakurai 1987; see also De Schutter 1995 for theoretical necessity). In addition, if the inhibitory interneuron activity coincides with the climbing-fiber activity, rebound potentiation (RP) is in- 
A

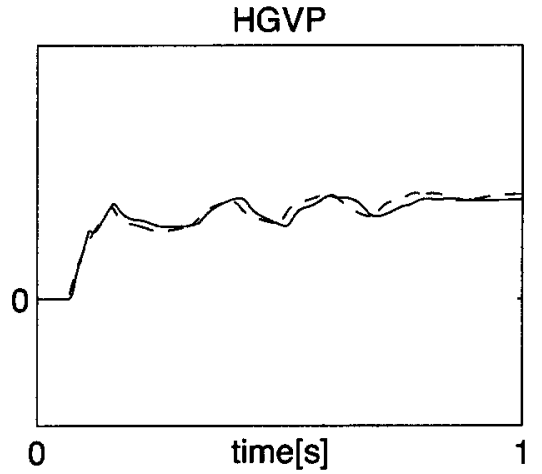

PVP

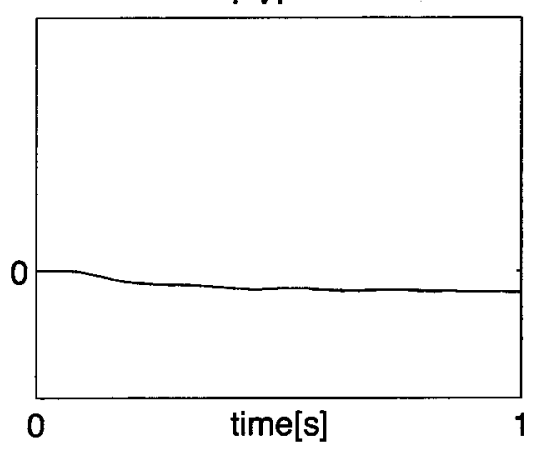

B
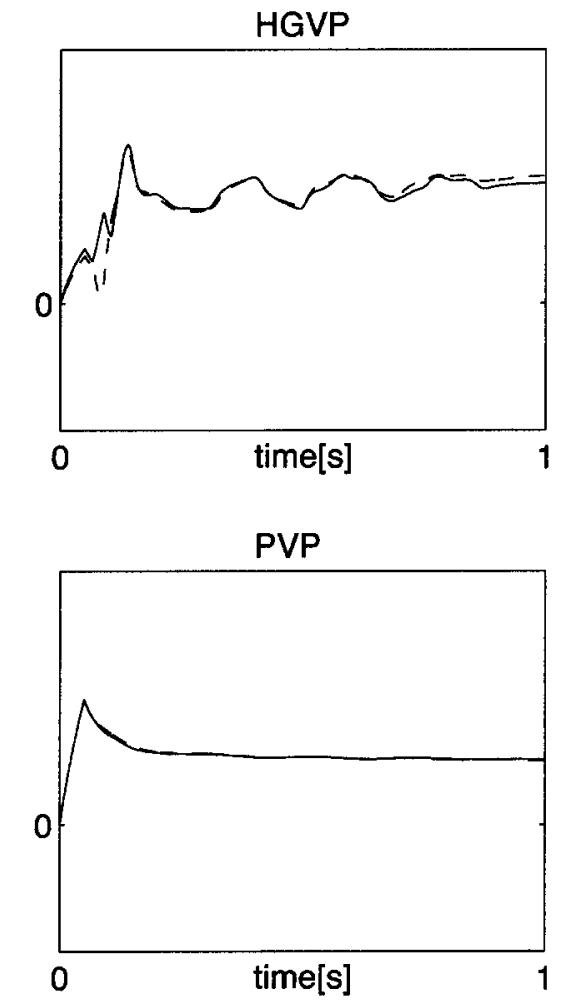

FTN
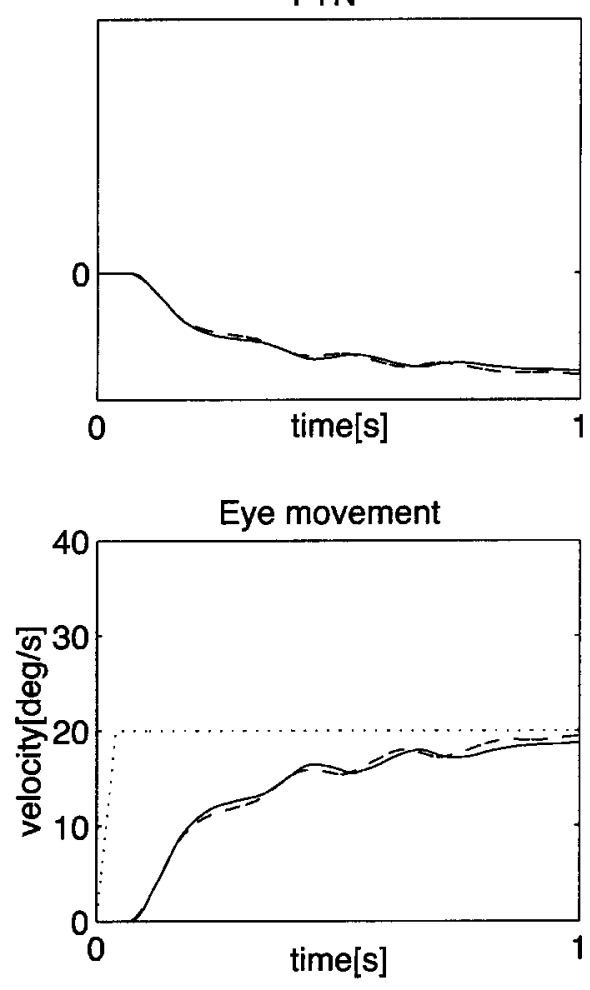

FTN

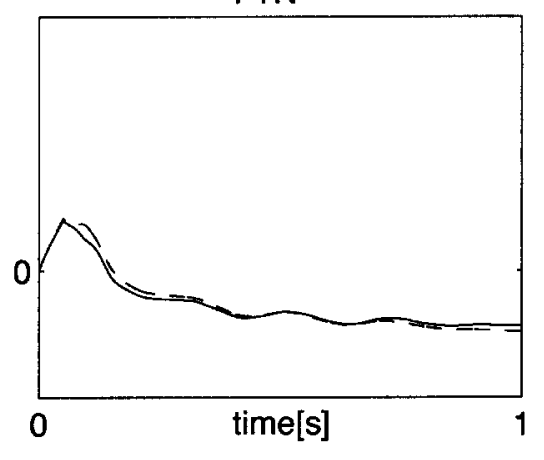

Eye movement

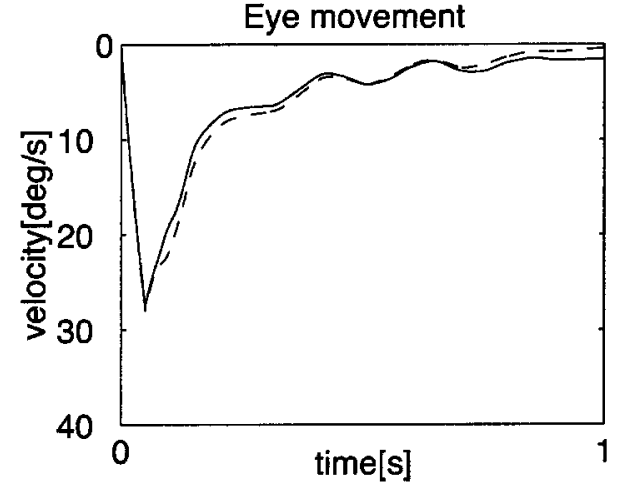

FIG. 5. A: simulation results of smooth pursuit in the new model. The neural activity of HGVP, flocculus target neurons (FTN) and position vestibular pause cells (PVP) and eye movement are shown when the visual target was moved on a ramp with the steadystate velocity of $20 \%$ s. B: simulation results of VOR cancellation. Both target velocity and head velocity are $20 \%$ s. - - , the results of Lisberger's (1994) model. The new model generated shows results almost identical to those of Lisberger's (1994) model. duced (Kano 1996; Kano et al. 1992). RP increases the effect of the inhibitory interneurons on the Purkinje cells.

The strength of the synaptic plasticity depends on the timing of the parallel- and climbing-fiber activity. This characteristic is called the temporal window of the plasticity. In the present study, we use a temporal window that peaks when the parallelfiber activity is $250 \mathrm{~ms}$ earlier than the climbing-fiber activity and that has $1 / 3$ of this peak when the parallel fiber activity is 
A
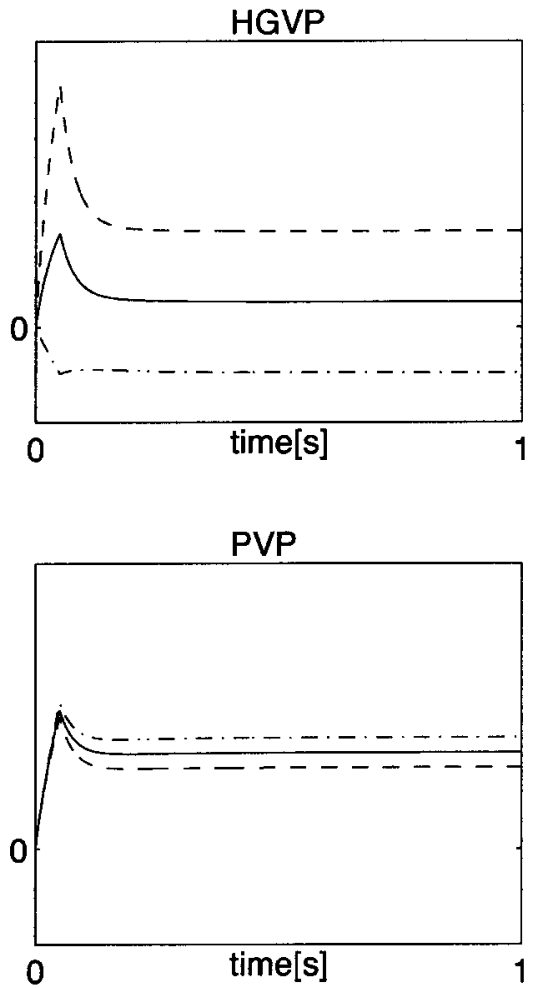

B
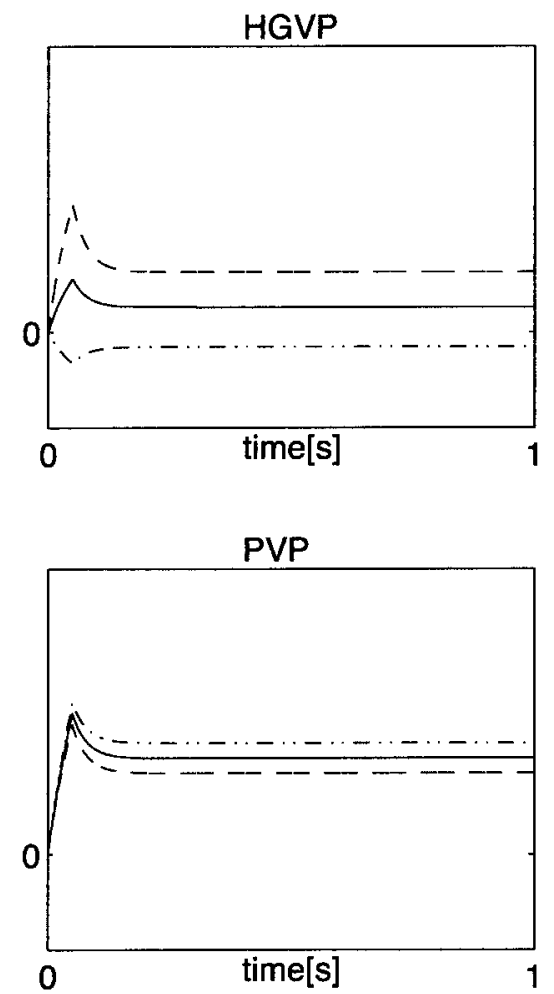

FTN
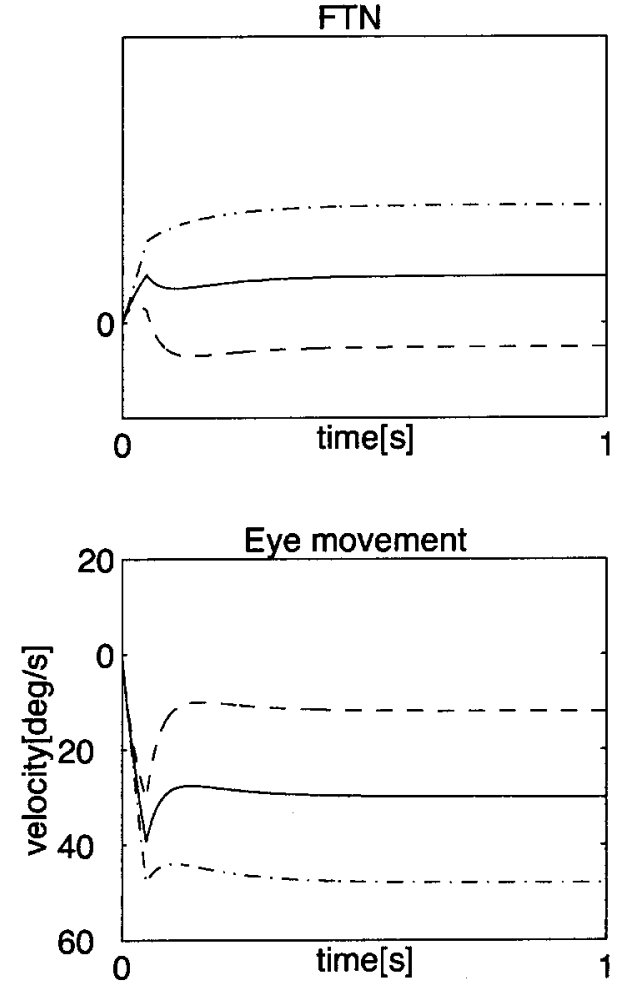

FTN
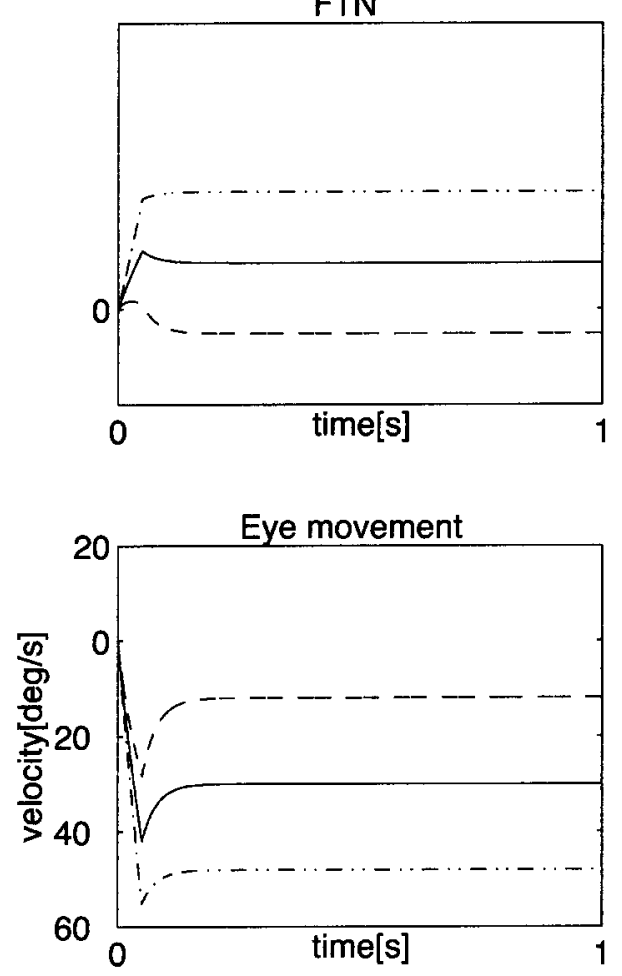

FIG. 6. Simulation results of VOR adaptation with hand-tuned synaptic weights. Neural activity of HGVP, FTN, and PVP and eye movement induced by ipsiversive head motions. - - -, - , and - - , low, normal, and high gains of VOR, respectively. A: simulation results of VOR adaptation in our new model. $B$ : simulation results of VOR adaptation in Lisberger's (1994) model. The new model can reproduce almost the same results as those of Lisberger's (1994) model.
$100 \mathrm{~ms}$ earlier than the climbing-fiber activity based on the study of Chen and Thompson (1995); this is a Gaussian distribution of SD $=85 \mathrm{~ms}$. This is also qualitatively consistent with the reinterpretation of a study on the LTD temporal window (Karachot et al. 1994) in Yamamoto et al. (2002; see also De Schutter 1995).
Yamamoto et al. (2002) derived the following learning equations, which incorporate the temporal window of the synaptic plasticity in the cerebellar cortex. The authors showed that OFR adaptation can be reproduced by these learning equations. Accordingly, we adopt them for the learning simulation of VOR in this paper. 


$$
\begin{aligned}
\eta(t) & =\int_{0}^{t} \operatorname{PF}(s) G(s-t) \mathrm{d} s \\
\tau \frac{\mathrm{d} w(t)}{\mathrm{d} t}= & -w(t) \\
& -\epsilon_{\mathrm{LTD}} \eta(t) 1\left(\mathrm{CS}(t)-\mathrm{CS}_{\mathrm{SP}}\right) \\
& +\epsilon_{\mathrm{LTP}} \eta(t) 1\left(\mathrm{CS}_{\mathrm{SP}}-\mathrm{CS}(t)\right)
\end{aligned}
$$

al. 1998). The reconstruction by such a generalized linear model of only the velocity of retinal image motion is statistically satisfactory (Yamamoto et al. 2002). Therefore we reconstruct $\operatorname{CS}(t)$ from the retinal slip signal with the generalized linear model.

$$
\mathrm{CS}(t)=S(0.0139 \cdot \dot{r}-6.866)
$$

where

$$
S(x)=\frac{\exp x}{1+\exp x}
$$

$\dot{r}$ denotes the retinal slip.

In monkey experiments using a combination of a rotating chair and a moving visual pattern on a screen, VOR adaptation can be induced in a few hours. In the present simulations, we set the learning coefficient appropriately to satisfy the results of monkey behavioral experiments, where $1 \mathrm{~h}$ of learning changes the VOR gain about 0.2 (Watanabe 1985). We set the learning coefficients $\epsilon_{\mathrm{LTD}}=\epsilon_{\mathrm{LTP}}$ to $2.10 \times 10^{-2}, 2.34 \times 10^{-3}$, and $0.70 \times 10^{-3}$, for $\mathrm{p} 1, \mathrm{p} 2$, and $\mathrm{e} 1$, respectively.

Figure 7 shows the results before and after learning for the VOR gain increase or decrease simulations. The case of the VOR gain $=1$ is shown as - . The results after the increase of the VOR gain are shown as - - -, and the results after the decrease of the VOR gain are shown as - - -. The VOR gain increased to 1.186 and decreased to 0.718 .

Table 1 shows the synaptic weight changes before and after learning simulations. We show the values used in the preceding hand-tuning simulations in parentheses. After the learning where the VOR gain increase had finished, the synaptic weight p1 and p2 inputs decreased and e1 increased by LTD and RP. The reason for the e 1 increase is given in the DISCUSSION. All of frequency of the complex spike at time $t, \operatorname{CS}(t)$ (Kobayashi et

HGVP

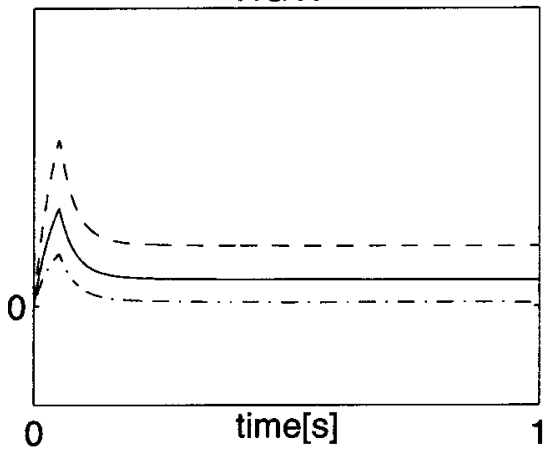

PVP

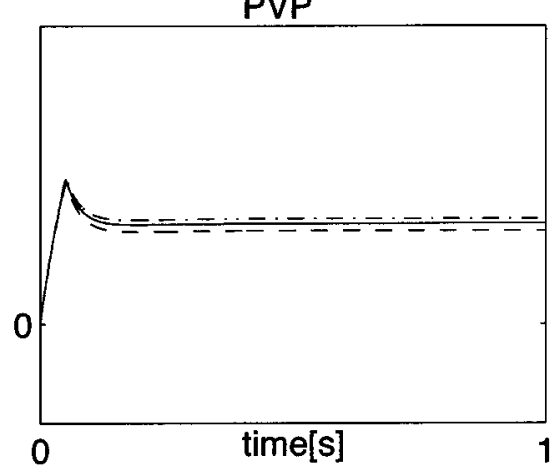

FTN
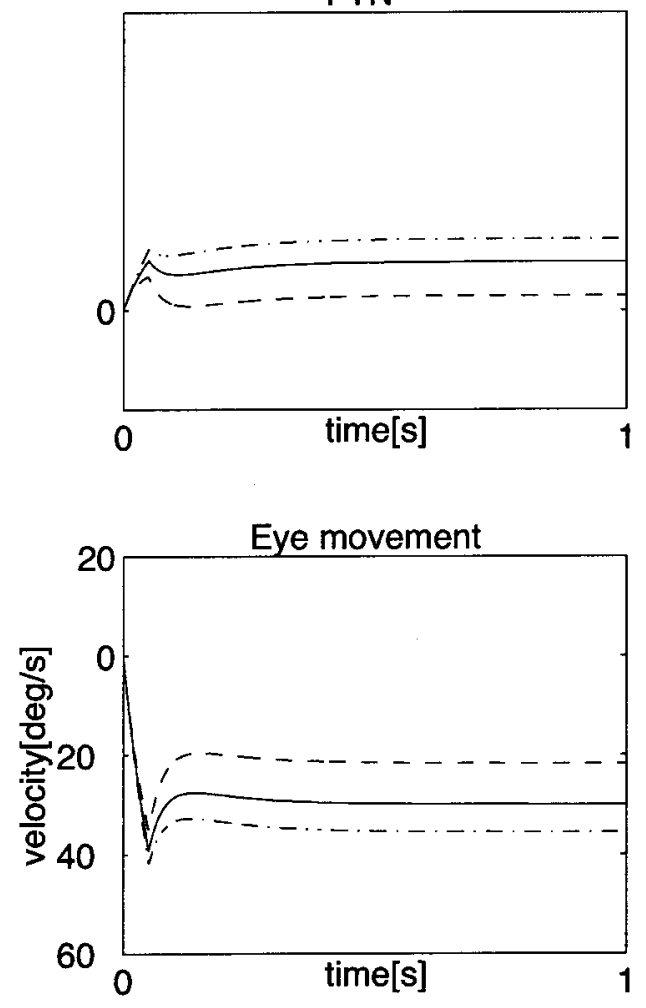

FIG. 7. Simulation results of VOR adaptation while using a differential equation model of the heterosynaptic plasticity of Purkinje cells. The solid line shows the case of the VOR gain $=1 .-\cdot$, the results after an increase in the VOR gain for learning; - --, the results after a decrease in the VOR gain for learning. These results are qualitatively similar to those in Fig. 6. 
the synaptic weights $\mathrm{p} 1, \mathrm{p} 2$ and e1 contributed to decreasing the HGVP activity (see Fig. $2 E$ to see the opposite polarity of p1, p2 inputs and e1 input). After learning where the VOR gain decrease had finished, all of the synaptic weights contributed to increasing the HGVP activity. These results are also consistent with the flocculus hypothesis.

We performed further simulations using a sine wave as a vestibular stimulus instead of a step-like function. Table 2 shows the results of the synaptic weight changes. In the sine wave simulations, our model could also reproduce VOR adaptation, as in the case of using a step-like stimulus as the vestibular inputs. We emphasize that the synaptic plasticity model formulated by Eqs. 8 and 9 can automatically reproduce VOR adaptation and furthermore automatically reproduce the expected directions of the change of p1, p2, and e1 based only on the interactions of the parallel- and climbing fiber inputs.

\section{I S C U S S I O N}

In summary, the parallel control-pathway theory was proposed on the grounds of considerable experimental data, including MST data during smooth-pursuit blink and OFR blank experiments. We assumed that the pursuit signal is maintained in the MST area, and mathematically demonstrated that the parallel control-pathway theory can reproduce the data of Purkinje cell activity in Miles et al. (1980b) at least equally as well as the gaze-velocity theory. Furthermore, we showed that a computational model with the heterosynaptic plasticity rule in the cerebellar cortex alone can stably reproduce VOR adaptation data. Our results demonstrate that the argument about the learning site of VOR adaptation critically depends on where the pursuit driving command is maintained.

\section{Site of the feedback loop maintaining the pursuit driving command}

In the rabbit, eye velocity is not a major factor in the floccular Purkinje cells (Miyashita and Nagao 1984; Nagao 1990, 1991). Here, we discuss whether or not the Purkinje cells in the flocculus and ventral paraflocculus in the monkey receive strong eye-velocity feedback.

In Lisberger (1994), it is stated that the site of the feedback loop maintaining the pursuit driving command from the vestibular nucleus to the cerebellar cortex is consistent with the following evidence. First, lesions of the flocculus and ventral paraflocculus cause severe but incomplete deficits in smooth pursuit (Zee et al. 1981). Second, mossy fibers transmit signals

TABLE 1. Synaptic weight changes before and after VOR adaptation while using a differential equation model of the heterosynaptic plasticity of Purkinje cells

\begin{tabular}{cccc}
\hline \hline VOR Gain & $\mathrm{p} 1$ & $\mathrm{p} 2$ & $\mathrm{e} 1$ \\
\hline$(1.6)$ & $(-0.0040)$ & $(-0.0200)$ & $(0.1850)$ \\
1.186 & 0.2250 & 0.0101 & 0.1801 \\
1 & 0.3300 & 0.0200 & 0.1750 \\
0.718 & 0.4936 & 0.0380 & 0.1727 \\
$(0.4)$ & $(0.6960)$ & $(0.0400)$ & $(0.1650)$ \\
\hline
\end{tabular}

The values used in hand-tuning simulations are shown in parentheses. The synaptic weight changes after learning are in the same direction as those of the hand-tuning values. VOR, vestibuloocular reflex.
TABLE 2. Synaptic weight changes before and after VOR adaptation for a sine wave stimulus

\begin{tabular}{lccc}
\hline \hline VOR Gain & $\mathrm{p} 1$ & $\mathrm{p} 2$ & $\mathrm{e} 1$ \\
\hline$(1.6)$ & $(-0.0040)$ & $(-0.0200)$ & $(0.1850)$ \\
1.203 & 0.2220 & 0.0028 & 0.1806 \\
1 & 0.3300 & 0.0200 & 0.1750 \\
0.807 & 0.4414 & 0.0332 & 0.1739 \\
$(0.4)$ & $(0.6960)$ & $(0.0400)$ & $(0.1650)$ \\
\hline
\end{tabular}

related to eye movements to the flocculus and ventral paraflocculus during both pursuit and VOR (Lisberger and Fuchs 1978; Miles et al. 1980b; Noda and Suzuki 1979; Stone 1987). Third, the discharge of HGVP cells related to eye velocity during pursuit is maintained during target stabilization in smooth pursuit (Stone and Lisberger 1990). Fourth, stimulation in the flocculus and ventral paraflocculus causes smooth eye movement (Belknap and Noda 1987; Lisberger 1994; Ron and Robinson 1973). Finally, smooth pursuit is maintained even when retinal slip signals are eliminated (Morris and Lisberger 1987). The explanation for this requires a pursuit-drivingcommand memory mechanism such as that provided by the positive feedback loop.

The evidence provided by the first and fourth items demonstrates that the flocculus and ventral paraflocculus are used for smooth pursuit. The evidence provided by the third and fifth items suggests the necessity of a mechanism that can maintain pursuit driving command independent of retinal slip signals. The second item indicates that eye-movement information is fed back to the flocculus and ventral paraflocculus. However, we do not think that any of this evidence directly supports the idea that the main mechanism for maintaining pursuit driving command exists between the cerebellar cortex and the vestibular nucleus. Furthermore, our model is consistent with all of this evidence.

The proposed pursuit-driving-command maintenance mechanism within MST works during zero-retinal slip pursuit as well as during pursuit blink but should not work for the OFR blank. There are at least two possible neural mechanisms for this. One is a switch that is on and off controlled by the attention required for smooth pursuit. Smooth pursuit eye movement is a voluntary movement, which requires attention on a target, while OFR is a reflex movement. Some brain areas outside the MST area, such as the frontal eye field, may influence the pursuit driving command maintenance mechanism within the MST area based on the level of attention. The other possible neural mechanism is related to the differences in visual stimuli for smooth pursuit and OFR. We assume a neural field that has receptive fields with different retinal locations. The neurons in the neural field are recurrently connected with short-range excitatory and long-range inhibitory connections. Erickson and Thier (1991) showed that many MSTd neurons decreased sensitivity to visual stimuli in the background during smooth pursuit. This result is consistent with the proposed lateral inhibition of MST neurons with different receptive field positions. During the smooth pursuit maintenance phase, a foveated small target excites a small number of neurons whose receptive field is around the fovea. Under this condition, the lateral inhibition does not operate because the visual stimulus is small and the self- 
excitation dominates. Therefore this structure could work as a short-term memory (i.e., the positive feedback loop with gain $m$ in Fig. 4). Thus even without retinal slip, the MST neurons can maintain their activity for a while. On the other hand, in OFR, a large-field stimulus motion excites many neurons whose receptive fields are spread over the visual field. Under this condition, lateral inhibition is dominant because many neurons are simultaneously excited initially by the large stimulus, and the positive feedback loop, due to the self-excitation, does not operate effectively. Thus MST activity quickly dies out when the stimulus is blanked. We have already confirmed that an MST neural field model can reproduce these expected differential behaviors for smooth pursuit blink and OFR blank (Tabata et al. 2001). Furthermore, the eye movement after the elimination of OKN stimulus can be explained based on the same framework.

Smooth pursuit also exhibits some predictive capabilities. For example, repetitive target motion every few seconds leads to predictive acceleration before the target begins to move (Wells and Barnes 1999). The MST area may also compute the information necessary for this kind of predictive smooth pursuit using recurrent neural connections.

\section{Synaptic weight changes of the vestibular inputs to Purkinje cells}

The gaze-velocity theory predicts that the sensitivity of HGVP to the vestibular inputs increases when the VOR gain increases and correspondingly decreases when the VOR gain decreases (Lisberger et al. 1994a; Miles et al. 1980a). This direction of change is exactly opposite to that predicted by the flocculus hypothesis (Ito 1972). This drastic difference is also evident in the two computer simulations conducted here. In Lisberger's (1994) model, p1 and p2 in Fig. 3 are used to determine the ratio between tonic responses and phasic-tonic responses, and p3 shows the weight of the vestibular inputs to the cerebellar cortex. p3 increases with a higher VOR gain and decreases with a lower VOR gain. On the other hand, in our new model, the weight of the vestibular inputs to the cerebellar cortex, p1 and p2 in Fig. 4, decreases with a higher VOR gain and increases with a lower VOR gain. Taking into consideration the sign inversion due to the Purkinje cell inhibition on its target neurons, the synaptic plasticity in the cerebellar cortex is the only mechanism to induce VOR adaptation in our model. However, it is against the main learning mechanism within the vestibular nucleus for VOR adaptation in Lisberger's (1994) model. We also note that the change of the positive feedback loop gain e1, automatically learned by the synaptic plasticity, is in the correct direction to drive the VOR adaptation in our model. The direction of e1 change is opposite to those of $\mathrm{p} 1$ and p2 because their parallel-fiber inputs possess the opposite polarity (see Fig. 2E), and learning Eqs. 8 and 9 induced the opposite change of direction.

This marked difference in the two models is almost directly derived from different interpretations of the origin of Purkinje cell activities during VOR cancellation. The gaze-velocity theory postulates that the HGVP-cell activity during VOR cancellation reflects the effect of the vestibular inputs because the HGVP cells encode the gaze velocity and the eye movement signal is zero during VOR cancellation (Fig. 2B). However, the parallel control-pathway theory postulates that the HGVP-cell activity during VOR cancellation is mainly generated by the estimated target-velocity signal coming down from the visual system (Fig. 2F). Our simulation results for VOR cancellation demonstrated that the simple positive feedback loop within the MST area shown in Fig. 4 can maintain the target velocity information with zero retinal slip smooth pursuit as well as during the steady-state phase of VOR cancellation. Therefore we demonstrate that the recording from HGVP cells during VOR cancellation by itself cannot uniquely determine the effect of changes in the VOR gain on the strength of the vestibular inputs to Purkinje cells.

Furthermore, our model reproduces HGVP activity after the VOR gain changes that is just like Lisberger's model (data not shown) when the sine-wave input is used in a similar manner to the physiological experiments (Miles et al. 1980a).

\section{Proposed experiments}

We now propose the following experiments to objectively examine the different predictions made by the current model and the gaze velocity theory.

In the first experiment, we suggest to record the neural activity from the same MST neuron after the visual stimulus is eliminated at the same point in time during OFR, pursuit, and VOR cancellation. It is predicted that the neural activity in the OFR case will decay much more rapidly than those in the pursuit and VOR cancellation cases. Furthermore, recording from an MT neuron under all conditions should result in a rapid decrease in the neural activity. These results would then indicate that the MST area is the major site of the mechanism maintaining target velocity during pursuit and VOR cancellation.

Second, we propose an experiment in which we observe eye movements and the activity of the MST neurons in total darkness after the target is suddenly eliminated during the steady-state phase of VOR cancellation. In the gaze-velocity theory, such target elimination should have no effect on eye movement, the neural activities of the MST area, or the cerebellar or vestibular neurons because Purkinje cells encode purely vestibular signals and have nothing to do with the visual input of the zero-retinal slip during VOR cancellation. On the other hand, our hypothesis predicts that the MST activity will first gradually decrease and then VOR will gradually be brought back through the visual-condition change from VOR cancellation to total darkness because Purkinje cells receive not only vestibular signals but also visual-related inputs vigorously. This prediction is at least consistent with the following physiological data (Kawano et al. 1984). Modulation in the activity of the visual tracking neurons in the MST area is negligible during VOR in the dark, while the visual tracking neurons are excited vigorously during VOR cancellation.

We thank Dr. K. Kawano of the National Institute of Advanced Industrial Science and Technology for useful comments, M. Namba of ATR for secretarial assistance, and Prof. H. Nishitani of the Nara Institute of Science and Technology for encouragement.

\section{REFERENCES}

BECKER W AND Fuch AF. Prediction in the oculomotor system: smooth pursuit during transient disappearance of a visual target. Exp Brain Res 57: 562575,1985 . 
BelKnAP DB AND NodA H. Eye movements evoked by microstimulation in the flocculus of the alert macaque. Exp Brain Res 67: 352-362, 1987.

Boussaoud D, Desimone R, AND UnGerleider LG. Subcortical connections of visual areas MST and FST in macaques. Vis Neurosci 9: 291-302, 1992.

BRODAL P. The corticopontine projection in the rhesus monkey: origin and principles of organization. Brain 101: 251-283, 1978.

BRODAL P. The pontocerebellar projection in the rhesus monkey: an experimental study with retrograde axonal transport of horseradish peroxidase. Neuroscience 4: 193-208, 1979.

BRODAL P. Further observations on the cerebellar projections from the pontine nuclei and the nucleus reticularis tegmenti pontis in the rhesus monkey. J Comp Neurol 204: 44-55, 1982.

Chen C And Thompson RF. Temporal specificity of long-term depression in parallel fiber-Purkinje synapses on rat cerebellar slice. Learn Mem 2: 185$198,1995$.

De SchutTer E. Cerebellar long-term depression might normalize excitation of Purkinje cells: a hypothesis. Trends Neurosci 18: 291-295, 1995.

DÜRSTELER MR AND WuRTZ RH. Pursuit optokinetic deficits following chemical lesions of cortical areas MT and MST. J Neurophysiol 60: 940-965, 1988.

ERICKSON RG AND Dow BM. Foveal tracking cells in the superior temporal sulcus of the macaque monkey. Exp Brain Res 78: 113-131, 1989.

ERICKSON RG AND THIER P. A neuronal correlate of spatial stability during periods of self-induced visual motion. Exp Brain Res 86: 608-616, 1991.

Fujita M. Adaptive filter model of the cerebellum. Biol Cybern 45: 195-206, $1982 \mathrm{a}$.

FujiTA M. Simulation of adaptive modification of the vestibuloocular reflex with an adaptive filter model of the cerebellum. Biol Cybern 45: 207-214, $1982 b$.

Glickstein M, Cohen JL, Dixon B, Gibson A, Hollins M, Labossiere E, AND RoBInson F. Corticopontine visual projections in macaque monkeys. J Comp Neurol 190: 209-229, 1980.

Glickstein M, May J, And Mercer BE. Corticopontine projection in the macaque: the distribution of labeled cortical cells after large injections of horse-radish peroxidase in the pontine nuclei. J Comp Neurol 235: 343-359, 1985.

GroH JM, Born RT, AND Newsome WT. How is a sensory map read out? Effects of microstimulation in visual area MT on saccades and smooth pursuit eye movements. J Neurosci 17: 4312-4330, 1997.

HIRANO T. Depression and potentiation of the synaptic transmission between a granule cell and a Purkinje cell in rat cerebellar culture. Neurosci Lett 119: 141-144, 1990.

Iто M. Neurophysiological aspects of the cerebellar motor control system. Int J Neurol 7: 162-176, 1970.

Iто M. Neural design of the cerebellar motor control system. Brain Res 40: 80-84, 1972.

Iто M. The Cerebellum and Neural Control. New York: Raven, 1984.

Iто M. Cerebellar flocculus hypothesis. Nature 363: 24-25, 1993.

Iто M. Cerebellar learning in the vestibuloocular reflex. Trends Cognit Sci 2: 313-321, 1998.

ITO M AND Kano M. Long-lasting depression of parallel fiber-Purkinje cell transmission induced by conjunctive stimulation of parallel fibers and climbing fibers in the cerebellar cortex. Neurosci Lett 33: 253-258, 1982.

Ito M, SAKURAi M, AND TONGROACH P. Climbing-fiber-induced depression of both mossy fiber responsiveness and glutamate sensitivity of cerebellar Purkinje cells. J Physiol (Lond) 324: 113-134, 1982.

KANO M. Long-lasting potentiation of GABAergic inhibitory synaptic transmission in cerebellar Purkinje cells: its properties and possible mechanisms. Behav Brain Sci 19: 354-361, 1996.

Kano M, Rexhausen U, Dreessen J, and Konnerth A. Synaptic excitation produces a long-lasting rebound potentiation of inhibitory synaptic signals in cerebellar Purkinje cells. Nature 356: 601-604, 1992.

KARACHOT L, KADO RT, AND ITO M. Stimulus parameters for induction of long-term depression in in vitro rat Purkinje cells. Neurosci Res 21: 161168, 1994.

KAWANO K. Ocular tracking: behavior and neurophysiology. Curr Opin Neurobiol 9: 467-473, 1999.

KawAno K, SASAKI M, And Yamashita M. Response properties of neurons in posterior parietal cortex of monkey during visual-vestibular stimulation. I. Visual tracking neurons. J Neurophysiol 51: 340-351, 1984.

KAWANO K AND ShIDARA M. The Role of the Ventral Paraflocculus in Ocular Following in the Monkey. Amsterdam: Elsevier, 1993, p. 195-202.
KaWAno K, Shidara M, Watanabe Y, and Yamane S. Neural activity in cortical area of MST of alert monkey during ocular following responses. J Neurophysiol 71: 2305-2324, 1994.

KAWANO K, ShIDARA M, AND YAMANE S. Neural activity in dorsolateral pontine nucleus of alert monkey during ocular following responses. $\mathrm{J} \mathrm{Neu}$ rophysiol 67: 680-703, 1992.

Kawato M AND Gomi H. The cerebellum and VOR/OKR learning models. Trends Neurosci 15: 445-453, 1992.

Kobayashi Y, Kawano K, Takemura A, Inoue Y, Kitama T, Gomi H, and Kawato M. Temporal firing patterns of Purkinje cells in the cerebellar ventral paraflocculus during ocular following responses in monkeys. II. Complex spikes. J Neurophysiol 80: 832-848, 1998.

Komatus H AND WuRTz RH. Modulation of pursuit eye movements by stimulation of cortical areas MT and MST. J Neurophysiol 62: 31-47, 1989.

KrauZlis RJ AND Lisberger SG. A control systems model of smooth pursuit eye movements with realistic emergent properties. Neural Comp 1: 116$122,1989$.

Langer T, Fuchs AF, Scudder CA, AND ChubB MC. Afferent to the flocculus of the cerebellum in the Rhesus macaque as revealed by retrograde transport of horseradish peroxidase. J Comp Neurol 235: 1-25, 1985.

LISBERGER SG. The neural basis for learning of simple motor skills. Science 242: $728-735,1988$.

LISBERGER SG. Neural basis for motor learning in the vestibuloocular reflex of primates. III. Computational and behavioral analysis of the sites of learning. J Neurophysiol 72: 974-998, 1994.

LisBeRGER SG AND FUCHS AF. Role of primate flocculus during rapid behavioral modification of vestibuloocular reflex. II. Mossy fiber firing patterns during horizontal head rotation and eye movement. J Neurophysiol 41: 764-777, 1978.

Lisberger SG AND PAVElKo TA. Vestibular signals carried by pathways subserving plasticity of the vestibuloocular reflex in monkeys. J Neurosci 6 : 346-354, 1986.

Lisberger SG, Pavelko TA, Bronte-Stewart HM, and Stone LS. Neural basis for motor learning in the vestibuloocular reflex of primates. II. Changes in the responses of horizontal gaze velocity Purkinje cells in the cerebellar flocculus and ventral paraflocculus. J Neurophysiol 72: 954-973, 1994a.

Lisberger SG, PAvelko TA, AND Broussard DM. Responses during eye movements of brain stem neurons that receive monosynaptic inhibition from the flocculus and ventral paraflocculus in monkeys. J Neurophysiol 72: 909-927, 1994b.

Lisberger SG, Pavelko TA, AND Broussard DM. Neural basis for motor learning in the vestibuloocular reflex of primates. I. Changes in the responses of brain stem neurons. J Neurophysiol 72: 928-953, 1994c.

LISBERGER SG AND SEJNOWSKI TJ. Motor learning in a recurrent network model based on the vestibuloocular reflex. Nature 360: 159-161, 1992.

MAUNSELL LHR AND VAN ESSEN DC. The connections of the middle temporal visual area (MT) and their relationship to a cortical hierarchy in the macaque monkey. J Neurosci 3: 2563-2586, 1983.

MAY JG AND ANDERSEN RA. Different patterns of corticopontine projections from separate cortical fields within the inferior parietal lobule and dorsal prelunate gyrus of the macaque. Exp Brain Res 63: 265-278, 1986.

MAy JG, Keller EL, AND SuZUKi DA. Smooth-pursuit eye movement deficits with chemical lesions in the dorsolateral pontine nucleus of the monkeys. J Neurophysiol 59: 952-977, 1988.

Miles FA, BRAITMAN DJ, AND Dow BM. Long-term adaptive changes in primate vestibuloocular reflex. IV. Electrophysiological observations in flocculus of adapted monkeys. J Neurophysiol 43: 1477-1493, 1980a.

Miles FA, Fuller JH, Braitman DJ, AND Dow BM. Long-term adaptive changes in primate vestibuloocular reflex. III. Electrophysiological observations in flocculus of normal monkeys. J Neurophysiol 43: 1437-1476, 1980 b.

Miles FA AND KAWANO K. Short-latency ocular following responses of monkey. III. Plasticity. J Neurophysiol 56: 1381-1396, 1986.

Miles FA, KAWANO K, AND OPTICAN LM. Short-latency ocular following responses of monkey. I. Dependence on temporospatial properties of visual input. J Neurophysiol 56: 1321-1354, 1986.

Miles FA AND LisBerger SG. Plasticity in the vestibuloocular reflex: a new hypothesis. Annu Rev Neurosci 4: 273-299, 1981.

MiYashita Y AND Nagao S. Analysis of signal content of Purkinje cells responses to optokinetic stimuli in the rabbit cerebellar flocculus by selective lesions of brain stem pathway. Neurosci Res 1: 223-241, 1984. 
MoRRIS EJ AND LISBERGER SG. Different responses to small visual errors during initiation and maintenance of smooth pursuit eye movements in monkeys. J Neurophysiol 58: 1351-1369, 1987.

NAGAO S. Eye velocity is not the major factor that determines mossy fiber responses of rabbit floccular Purkinje cells to head and screen oscillation. Exp Brain Res 80: 221-224, 1990.

NAGAO S. Contribution of oculomotor signals to the behavior of rabbit floccular Purkinje cells during reflex eye movements. Neurosci Res 12: 169184, 1991.

Newsome WT, Wurtz RH, And Komatsu H. Relation of cortical areas MT and MST to pursuit eye movements. II. Differentiation of retinal from extraretinal inputs. J Neurophysiol 60: 604-620, 1988.

NodA H AND SuzuKi DA. The role of flocculus of the monkey in fixation and smooth pursuit eye movements. J Physiol (Lond) 294: 335-348, 1979.

POLA J AND WyatT HJ. Offset dynamics of human smooth pursuit eye movements: effects of target presence and subject attention. Vision Res 37: 2579-2595, 1997.

RON S AND ROBINSON DA. Eye movements evoked by cerebellar stimulation in the alert monkey. J Neurophysiol 36: 1004-1021, 1973.

Sakata H, Shibutani H, and Kawano K. Functional properties of visual tracking neurons in posterior parietal association cortex of the monkey. J Neurophysiol 49: 1364-1380, 1983.

SAKURAi M. Synaptic modification of parallel fibre-Purkinje cell transmission in in vitro guinea pig cerebellar slices. J Physiol (Lond) 394: 463-480, 1987.

SHIDARA M AND KAWANO K. Role of Purkinje cells in the ventral paraflocculus in short-latency ocular following responses. Exp Brain Res 93: 185-195, 1993.

Shidara M, KaWAno K, And Yamane S. Ocular following response deficits with chemical lesions in the medial superior temporal area of the monkey (Abstract). Neurosci Res 14: S69, 1991.

Stone LS. Visual Simple-Spike and Complex-Spike Responses of Gaze Velocity Purkinje Cells in the Cerebellar Flocculus During Smooth Pursuit Eye
Movement in Monkeys (PhD dissertation). San Francisco, CA: Univ. of California, 1987.

StONE LS AND LISBERger SG. Visual responses of Purkinje cells in the cerebellar flocculus during smooth-pursuit eye movements in monkeys. I. Simple spikes. J Neurophysiol 63: 1241-1261, 1990.

Tabata H, Shibata T, Taguchi S, and Kawato M. Simulation study on the smooth pursuit eye movements with MST-neural field model. Tech Rep IEICE NC2000-101: 63-70, 2001.

Takemura A, Inoue K, and Kawano $\mathrm{K}$. The role of MST neurons in short-latency visual tracking eye movements. J Soc Neurosci Abstr 26: $1715,2000$.

THIER P AND ERICKSON RG. Responses of visual-tracking neurons from cortical area MST-1 to visual, eye, and head motion. Eur J Neurosci 4: 539-553, 1992.

TUSA RJ AND UnGERLEIDER LG. Fiber pathways of cortical areas mediating smooth pursuit eye movements in monkeys. Ann Neurol 23: 174-183, 1988.

Ungerleider LG, Desimone R, Galkin TW, and Mishrin M. Subcortical projections of area MT in the macaque. J Comp Neurol 223: 368-386, 1984.

UNGERLEIDER LG AND MAUnSELl JHR. Cortical connections of visual area MT in the macaque. J Comp Neurol 248: 190-222, 1986.

WATANABE E. Role of primate flocculus in the adaptation of the vestibuloocular reflex. Neurosci Res 3: 20-28, 1985.

WeLls SG AND BARNES GR. Predictive smooth pursuit eye movements during identification of moving acuity targets. Vision Res 39: 2767-2775, 1999.

Yamamoto K, Kobayashi Y, Takemura A, Kawano K, and Kawato M. Computational studies on acquisition and adaptation of ocular following responses based on cerebellar synaptic plasticity. J Neurophysiol 87: 15541571, 2002.

Zee DS, YamazaKi A, Butler PH, AND Gucer G. Effects of ablation of the flocculus and paraflocculus on eye movements in the primate. $J$ Neurophysiol 46: 878-899, 1981. 